\title{
Positive periodic solution for $p$-Laplacian neutral Rayleigh equation with singularity of attractive type
}

\author{
Yun Xin ${ }^{1}$, Hongmin Liu ${ }^{1 *}$ and Zhibo Cheng ${ }^{2,3}$
}

${ }^{\text {*Correspondence: }}$
hongminliu_1982@126.com
${ }^{1}$ College of Computer Science and
Technology, Henan Polytechnic
University, Jiaozuo, China
Full list of author information is
available at the end of the article

\begin{abstract}
In this paper, we consider a kind of $p$-Laplacian neutral Rayleigh equation with singularity of attractive type,

$$
\left(\phi_{p}(u(t)-c u(t-\delta))^{\prime}\right)^{\prime}+f\left(t, u^{\prime}(t)\right)+g(t, u(t))=e(t) .
$$

By applications of an extension of Mawhin's continuation theorem, sufficient conditions for the existence of periodic solution are established.
\end{abstract}

Keywords: Neutral operator; $p$-Laplacian; Periodic solution; Rayleigh equation; Singularity of attractive type

\section{Introduction}

As is well known, the Rayleigh equation can be derived from many fields, such as physics, mechanics and engineering technique fields, and an important question is whether this equation can support periodic solutions. In 1977, Gaines and Mawhin [1] introduced some continuation theorems and applied this theorem to discussing the existence of solutions for the Rayleigh equation [1, p. 99]

$$
u^{\prime \prime}+f\left(u^{\prime}\right)+g(t, u)=0
$$

Gaines and Mawhin's work has attracted the attention of many scholars in the field of the Rayleigh equations. More recently, the existence of periodic solutions for Rayleigh equation was extensively studied (see [2-11] and the references therein). Some classical tools have been used to study Rayleigh equation in the literature, including the method of upper and lower solutions [6], the time map continuation theorem [7, 9], fixed point theory [4], the Manásevich-Mawhin continuation theorem [10,11], and coincidence degree theory $[2,3,5,8]$.

Recently there have been published some results on singular Rayleigh equations [1216]. In 2015, Wang and Ma [15] investigated the following singular Rayleigh equation:

$$
u^{\prime \prime}+f\left(t, u^{\prime}\right)+g(u)=p(t)
$$

(c) The Author(s) 2018. This article is distributed under the terms of the Creative Commons Attribution 4.0 International License (http://creativecommons.org/licenses/by/4.0/), which permits unrestricted use, distribution, and reproduction in any medium, provided you give appropriate credit to the original author(s) and the source, provide a link to the Creative Commons license, and indicate if changes were made. 
where $g$ had a repulsive singularity at the origin, i.e.,

$$
\lim _{u \rightarrow 0^{+}} g(u)=-\infty
$$

By applications of the limit properties of the time map, the authors obtained the result of the existence of periodic solution for this equation. Afterwards, by using topological degree theory, Chen and Lu [12] discussed that the existence of periodic solution for the following singular Rayleigh equations:

$$
u^{\prime \prime}+f\left(t, u^{\prime}\right)+\varphi(t) u(t)-\frac{1}{u^{r}(t)}=h(t) .
$$

The authors found new methods for estimating a lower priori bounds of periodic solutions to equation (1.2). Recently, Xin and Cheng [16] investigated a kind of neutral Rayleigh equation with singularity of repulsive type,

$$
(u(t)-c u(t-\delta))^{\prime \prime}+f\left(t, u^{\prime}(t)\right)+g(t, u(t))=e(t)
$$

where $g(t, u)=g_{1}(t, u)+g_{0}(u)$ and $g_{0}$ had a strong singularity at $u=0$, i.e.,

$$
\lim _{u \rightarrow 0^{+}} \int_{1}^{u} g_{0}(s) d s=+\infty
$$

By applications of coincidence degree theory, the authors found the existence of positive periodic solution for equation (1.3).

All the aforementioned results are related to Rayleigh equation or neutral Rayleigh equation with singularity of repulsive type. Naturally, a new question arises: how $p$-Laplacian neutral Rayleigh equation works on singularity of attractive type? Besides practical interests, the topic has obvious intrinsic theoretical significance. To answer this question, in this paper, we consider a kind of $p$-Laplacian neutral Rayleigh equation with singularity of attractive type,

$$
\left(\phi_{p}(u(t)-c u(t-\delta))^{\prime}\right)^{\prime}+f\left(t, u^{\prime}(t)\right)+g(t, u(t))=e(t)
$$

where $p>1, \varphi_{p}(u)=|u|^{p-2} u$ for $u \neq 0$ and $\varphi_{p}(0)=0 ;|c| \neq 1$ and $\delta$ is a constant with $0 \leq$ $\delta<\omega ; e: \mathbb{R} \rightarrow \mathbb{R}$ is continuous periodic functions with $e(t+\omega)-e(t) \equiv 0$ and $\int_{0}^{T} e(t) d t=$ 0 ; $f$ is for continuous functions defined on $\mathbb{R}^{2}$ and periodic in $t$ with $f(t, \cdot)=f(t+\omega, \cdot)$ and $f(t, 0)=0, g(t, u)=g_{0}(u)+g_{1}(t, u)$, here $g_{1}: \mathbb{R} \times(0,+\infty) \rightarrow \mathbb{R}$ is an $L^{2}$-Carathéodory function, $g_{1}(t, \cdot)=g_{1}(t+\omega, \cdot) ; g_{0} \in C((0, \infty) ; \mathbb{R})$ has an attractive singularity at the origin, i.e.,

$$
\lim _{u \rightarrow 0^{+}} \int_{1}^{u} g_{0}(s) d s=-\infty
$$

Obviously, the attractive condition (1.6) is in contradiction with the repulsive singularity of (1.1) and (1.4). Therefore, the above methods of $[12,15,16]$ are no long applicable to the proof of existence of a periodic solution for (1.5) with singularity of attractive type. So we need to find a new method to get over it. 
In this paper, by applications of an extension of Mawhin's continuation theorem in [17] and some analysis techniques, we see the existence of a positive periodic solution for (1.5). Our results improve and extend the results in $[12,15,16]$.

\section{Preliminary lemmas}

For convenience, define

$$
C_{\omega}^{1}=\left\{u \in C^{1}(\mathbb{R}, \mathbb{R}): u(t+\omega)=u(t)\right\},
$$

which is a Banach space endowed with the norm $\|\cdot\|$ define by $\|u\|=\max \left\{\|u\|_{\infty},\left\|u^{\prime}\right\|_{\infty}\right\}$, for all $x$, and

$$
\|u\|_{\infty}=\max _{t \in[0, \omega]}|u(t)|, \quad\left\|u^{\prime}\right\|_{\infty}=\max _{t \in[0, \omega]}\left|u^{\prime}(t)\right| .
$$

Lemma 2.1 (see [18]) If $|c| \neq 1$, then the operator $(A u)(t):=u(t)-c u(t-\delta)$ has a continuous inverse $A^{-1}$ on the space $C_{\omega}$, and satisfying

$$
\left(A^{-1} f\right)(t)= \begin{cases}f(t)+\sum_{j=1}^{\infty} c^{j} f(t-j \delta), & \text { for }|c|<1, \forall f \in C_{\omega}, \\ -\frac{f(t+\delta)}{c}-\sum_{j=1}^{\infty} \frac{1}{j^{+1}} f(t+(j+1) \delta), & \text { for }|c|>1, \forall f \in C_{\omega} .\end{cases}
$$

Lemma 2.2 If $|c| \neq 1$, then operator $A^{-1}$ satisfying

$$
\int_{0}^{\omega}\left|\left(A^{-1} f\right)(t)\right|^{p} d t \leq \frac{1}{|1-| c||^{p}} \int_{0}^{\omega}|f(t)|^{p} d t, \quad \forall f \in C_{\omega} \text {, here } 1 \leq p<\infty .
$$

Proof We first consider $|c|<1$. From Lemma 2.1, we have

$$
\begin{aligned}
\int_{0}^{\omega}\left|\left(A^{-1} f\right)(t)\right|^{p} d t & =\int_{0}^{\omega}\left|\sum_{j=0}^{\infty} c^{j} f(t-j \delta)\right|^{p} d t \\
& \leq \int_{0}^{\omega}\left(\sum_{j=0}^{\infty}\left|c^{j} f(t-j \delta)\right|\right)^{p} d t \\
& \leq \frac{1}{(1-|c|)^{p}} \int_{0}^{\omega}|f(t)|^{p} d t .
\end{aligned}
$$

Similarly, for $|c|>1$, we can get

$$
\int_{0}^{\omega}\left|\left(A^{-1} f\right)(t)\right|^{p} d t \leq \frac{1}{(|c|-1)^{p}} \int_{0}^{\omega}|f(t)|^{p} d t .
$$

Therefore, we have

$$
\int_{0}^{T}\left|\left(A^{-1} f\right)(t)\right|^{p} d t \leq \frac{1}{|1-| c||^{p}} \int_{0}^{T}|f(t)|^{p} d t .
$$

Lemma 2.3 (see [19]) If $u \in C_{\omega}^{1}(\mathbb{R}, \mathbb{R})$, and there exists a point $t^{*} \in[0, \omega]$ such that $\left|u\left(t^{*}\right)\right|<$ d, then

$$
\|u\|_{\infty} \leq d+\frac{1}{2} \int_{0}^{\omega}|u(t)|^{\prime} d t
$$


and

$$
\left(\int_{0}^{\omega}|u(t)|^{p} d t\right)^{\frac{1}{p}} \leq\left(\frac{\omega}{\pi_{p}}\right)\left(\int_{0}^{\omega}\left|u^{\prime}(t)\right|^{2} d t\right)^{\frac{1}{p}}+d \omega^{\frac{1}{p}}
$$

where $1 \leq p<\infty, \pi_{p}=2 \int_{0}^{(p-1) / p} \frac{d s}{\left(1-\frac{s^{p}}{p-1}\right)^{1 / p}}=\frac{2 \pi(p-1)^{1 / p}}{p \sin (\pi / p)}$.

The following lemma involves the consequences of Theorem 3.1 of [17].

Lemma 2.4 Assume that condition $|c| \neq 1, \Omega$ is an open bounded set in $C_{\omega}^{1}$. If:

(i) for each $\lambda \in(0,1)$ the equation

$$
\left(\phi_{p}(A u)^{\prime}(t)\right)^{\prime}+\lambda f\left(t, u^{\prime}(t)\right)+\lambda g(t, u(t))=\lambda e(t)
$$

has no solution on $\partial \Omega$;

(ii) the equation

$$
F(a):=\frac{1}{\omega} \int_{0}^{\omega} g(t, a) d t=0
$$

has no solution on $\partial \Omega \cap \mathbb{R}$;

(iii) the Brouwer degree

$$
\operatorname{deg}\{F, \Omega \cap \mathbb{R}, 0\} \neq 0,
$$

then Eq. (2.1) has at least one periodic solution on $\bar{\Omega}$.

\section{Main results: positive periodic solution for (1.5)}

In this section, we will consider the existence of a positive periodic solution for (1.5) with singularity.

Theorem 3.1 Assume that the following conditions hold:

$\left(H_{1}\right)$ there exists a positive constant $K$ such that $|f(t, v)| \leq K$, for $(t, v) \in \mathbb{R} \times \mathbb{R}$;

$\left(H_{2}\right)$ there exist positive constants $D_{1}$ and $D_{2}$ with $D_{1}>D_{2}>0$ such that $g(t, u)<-K$ for $(t, u) \in \mathbb{R} \times\left(D_{1},+\infty\right)$ and $g(t, u)>K$ for $(t, u) \in \mathbb{R} \times\left(0, D_{2}\right)$

$\left(H_{3}\right)$ there exist positive constants $a, b$ such that

$$
-g(t, u) \leq a u^{p-1}+b, \quad \text { for all } u>0
$$

Then (1.5) has at least one positive solution with period $\omega$ if $\frac{\omega(1+|c|)^{\frac{1}{p}} a^{\frac{1}{p}}}{|1-| c||}<2^{\frac{p-1}{p}}$.

Proof Firstly, we will claim that the set of all possible $\omega$-periodic solutions of (2.1) is bounded. Let $u(t) \in C_{\omega}^{1}$ be an arbitrary solution of (2.1) with period $\omega$.

We claim that there exists a point $t_{0} \in[0, \omega]$ such that

$$
0<u\left(t_{0}\right) \leq D_{1} .
$$


Integrating both sides of $(2.1)$ over $[0, \omega]$, we have

$$
\int_{0}^{\omega}\left[f\left(t, u^{\prime}(t)\right)+g(t, u(t))\right] d t=0 .
$$

Therefore, from $\left(H_{1}\right)$, we have

$$
-K \omega \leq \int_{0}^{\omega} g(t, u(t)) d t \leq K \omega .
$$

From $\left(H_{2}\right)$, we know that there exist two points $t_{0}, \tau \in(0, T)$, such that

$$
u\left(t_{0}\right) \leq D_{1}, \quad \text { and } \quad u(\tau)>D_{2} .
$$

Since $u(t)>0, t \in[0, \omega]$, we get $0<u\left(t_{0}\right) \leq D_{1}$. Equation (3.1) is proved.

Then, from Lemma 2.3, we have

$$
\|u\|_{\infty} \leq D_{1}+\frac{1}{2} \int_{0}^{\omega}\left|u^{\prime}(s)\right| d s .
$$

Multiplying both sides of $(2.1)$ by $(A u)(t)$ and integrating over $[0, \omega]$, we get

$$
\begin{aligned}
& \int_{0}^{\omega}\left(\phi_{p}(A u)^{\prime}(t)\right)^{\prime}(A u)(t) d t+\lambda \int_{0}^{\omega} f\left(t, u^{\prime}(t)\right)(A u)(t) d t+\lambda \int_{0}^{\omega} g(t, u(t))(A u)(t) d t \\
& \quad=\lambda \int_{0}^{\omega} e(t)(A u)(t) d t
\end{aligned}
$$

i.e.

$$
\begin{aligned}
\int_{0}^{\omega}\left|(A u)^{\prime}(t)\right|^{p} d t= & \lambda \int_{0}^{\omega} f\left(t, u^{\prime}(t)\right)(A u)(t) d t+\lambda \int_{0}^{\omega} g(t, u(t))(A u)(t) d t \\
& -\lambda \int_{0}^{\omega} e(t)(A u)(t) d t
\end{aligned}
$$

From $\left(H_{1}\right)$, we have

$$
\begin{aligned}
& \int_{0}^{\omega}\left|(A u)^{\prime}(t)\right|^{p} d t \\
& \quad \leq(1+|c|) \int_{0}^{\omega}\left|f\left(t, u^{\prime}(t)\right)\right||u(t)| d t+\int_{0}^{\omega}|g(t, u(t))||u(t)| d t+\int_{0}^{\omega}|e(t)||u(t)| d t \\
& \quad \leq(1+|c|)\|u\|_{\infty}\left(\int_{0}^{\omega}\left|f\left(t, u^{\prime}(t)\right)\right| d t+\int_{0}^{\omega}|g(t, u(t))| d t+\int_{0}^{\omega}|e(t)| d t\right) \\
& \quad \leq(1+|c|)\|u\|_{\infty}\left(K \omega+\|e\|_{\infty} \omega+\int_{0}^{\omega}|g(t, u(t))| d t\right) .
\end{aligned}
$$

We get from $\left(H_{1}\right),\left(H_{3}\right)$ and $(3.2)$

$$
\begin{aligned}
\int_{0}^{\omega}|g(t, u(t))| d t & =\int_{g(t, u(t)) \geq 0} g^{+}(t, u(t)) d t-\int_{g(t, u(t)) \leq 0} g^{-}(t, u(t)) d t \\
& =-2 \int_{g(t, u(t)) \leq 0} g^{-}(t, u(t)) d t+\int_{0}^{\omega} f\left(t, u^{\prime}(t)\right) d t
\end{aligned}
$$




$$
\begin{aligned}
& \leq 2 a \int_{0}^{\omega}|u(t)|^{p-1} d t+2 b \omega+K \omega \\
& \leq 2 a \omega\|u\|_{\infty}^{p-1}+2 b \omega+K \omega,
\end{aligned}
$$

where $g^{-}:=\min \{g(t, u), 0\}$. Substituting (3.4) and (3.7) into (3.6), we have

$$
\begin{aligned}
\int_{0}^{\omega}\left|(A u)^{\prime}(t)\right|^{p} d t \leq & (1+|c|)\|u\|_{\infty}\left(2 a \omega\|u\|_{\infty}^{p-1}+2 b \omega+2 K \omega+\|e\|_{\infty} \omega\right) \\
= & 2(1+|c|) a \omega\|u\|_{\infty}^{p}+(1+|c|) N_{1}\|u\|_{\infty} \\
\leq & 2(1+|c|) a \omega\left(D_{1}+\frac{1}{2} \int_{0}^{\omega}\left|u^{\prime}(t)\right| d t\right)^{p} \\
& +(1+|c|) N_{1}\left(D_{1}+\frac{1}{2} \int_{0}^{\omega}\left|u^{\prime}(t)\right| d t\right) \\
= & \frac{(1+|c|) a \omega}{2^{p-1}}\left(1+\frac{2 D_{1}}{\int_{0}^{\omega}\left|u^{\prime}(t)\right| d t}\right)^{p}\left(\int_{0}^{\omega}\left|u^{\prime}(t)\right| d t\right)^{p} \\
& +\frac{1}{2}(1+|c|) N_{1} \int_{0}^{\omega}\left|u^{\prime}(t)\right| d t+(1+|c|) N_{1} D_{1},
\end{aligned}
$$

where $N_{1}:=2 b \omega+2 K \omega+\|e\|_{\infty} \omega$. For a given constant $\zeta>0$, which is only dependent on $k>0$, we have

$$
(1+u)^{k} \leq 1+(1+k) u, \quad \text { for } u \in[0, \zeta] .
$$

Therefore, we have

$$
\begin{aligned}
\int_{0}^{\omega}\left|(A u)^{\prime}(t)\right|^{p} d t \leq & \frac{(1+|c|) a \omega}{2^{p-1}}\left(1+\frac{2 D_{1} p}{\int_{0}^{\omega}\left|u^{\prime}(t)\right| d t}\right)\left(\int_{0}^{\omega}\left|u^{\prime}(t)\right| d t\right)^{p} \\
& +\frac{1}{2}(1+|c|) N_{1} \int_{0}^{\omega}\left|u^{\prime}(t)\right| d t+(1+|c|) N_{1} D_{1} \\
= & \frac{(1+|c|) a \omega}{2^{p-1}}\left(\int_{0}^{\omega}\left|u^{\prime}(t)\right| d t\right)^{p}+\frac{(1+|c|) a \omega D_{1} p}{2^{p-2}}\left(\int_{0}^{\omega}\left|u^{\prime}(t)\right| d t\right)^{p-1} \\
& +\frac{1}{2}(1+|c|) N_{1} \int_{0}^{\omega}\left|u^{\prime}(t)\right| d t+(1+|c|) N_{1} D_{1}
\end{aligned}
$$

By application of Lemma 2.1, we have

$$
\begin{aligned}
\int_{0}^{\omega}\left|u^{\prime}(t)\right| d t & =\int_{0}^{\omega}\left|\left(A^{-1} A u^{\prime}\right)(t)\right| d t \\
& \leq \frac{\int_{0}^{\omega}\left|(A u)^{\prime}(t)\right| d t}{|1-| c||} \\
& \leq \frac{\omega^{\frac{1}{q}}\left(\int_{0}^{\omega}\left|(A u)^{\prime}(t)\right|^{p} d t\right)^{\frac{1}{p}}}{|1-| c||},
\end{aligned}
$$

since $\left(A u^{\prime}\right)(t)=(A u)^{\prime}(t)$ and $\frac{1}{p}+\frac{1}{q}=1$. Apply the inequality

$$
(a+b)^{k} \leq a^{k}+b^{k}, \quad \text { for } a, b>0,0<k<1 .
$$


Substituting (3.8) into (3.9), we have

$$
\begin{aligned}
\int_{0}^{\omega}\left|u^{\prime}(t)\right| d t \leq & \frac{\omega^{\frac{1}{q}}\left(\frac{(1+|c|) a \omega}{2^{p-1}}\right)^{\frac{1}{p}} \int_{0}^{\omega}\left|u^{\prime}(t)\right| d t+\omega^{\frac{1}{q}}\left(\frac{(1+|c|) a \omega D_{1} p}{2^{p-2}}\right)^{\frac{1}{p}}\left(\int_{0}^{\omega}\left|u^{\prime}(t)\right| d t\right)^{\frac{p-1}{p}}}{|1-| c||} \\
& +\frac{\omega^{\frac{1}{q}}\left(\frac{1}{2}(1+|c|) N_{1} \int_{0}^{\omega}\left|u^{\prime}(t)\right| d t\right)^{\frac{1}{p}}+\omega^{\frac{1}{q}}\left((1+|c|) N_{1} D_{1}\right)^{\frac{1}{p}}}{|1-| c||} .
\end{aligned}
$$

Since $\frac{\omega(1+|c|)^{\frac{1}{p}} a^{\frac{1}{p}}}{|1-| c||}<2^{\frac{p-1}{p}}$, it is easy to see that there exists a positive constant $M_{1}^{\prime}$ such that

$$
\int_{0}^{\omega}\left|u^{\prime}(t)\right| d t \leq M_{1}^{\prime}
$$

From (3.4) and (3.10), we have

$$
\|u\|_{\infty} \leq D_{1}+\frac{1}{2} \int_{0}^{\omega}\left|u^{\prime}(t)\right| d t \leq D_{1}+\frac{1}{2} M_{1}^{\prime}:=M_{1} .
$$

As $(A u)(0)=(A u)(\omega)$, there exists $t_{1} \in[0, \omega]$ such that $(A u)^{\prime}\left(t_{1}\right)=0$, while $\phi_{p}(0)=0$, we have

$$
\begin{aligned}
\left|\phi_{p}\left((A u)^{\prime}(t)\right)\right| & =\left|\int_{t_{1}}^{t}\left(\phi_{p}\left((A u)^{\prime}(s)\right)\right)^{\prime} d s\right| \\
& \leq \lambda \int_{0}^{\omega}\left|f\left(t, u^{\prime}(t)\right)\right| d t+\lambda \int_{0}^{\omega}|g(t, u(t))| d t+\lambda \int_{0}^{\omega}|e(t)| d t,
\end{aligned}
$$

where $t \in\left[t_{1}, t_{1}+\omega\right]$. In view of $\left(H_{1}\right),(3.7)$ and (3.12), we have

$$
\begin{aligned}
\left\|\phi_{p}(A u)^{\prime}\right\|_{\infty} & =\max _{t \in[0, \omega]}\left\{\left|\phi_{p}\left((A u)^{\prime}(t)\right)\right|_{\infty}\right\} \\
& =\max _{t \in\left[t_{1}, t_{1}+\omega\right]}\left\{\left|\int_{t_{1}}^{t}\left(\phi_{p}\left((A u)^{\prime}(s)\right)\right)^{\prime} d s\right|\right\} \\
& \leq \lambda\left(\int_{0}^{\omega} \mid f\left(t, u^{\prime}(t)\left|d t+\int_{0}^{\omega}\right| g(t, u(t))\left|d t+\int_{0}^{\omega}\right| e(t) \mid d t\right)\right. \\
& \leq \lambda\left(K \omega+2 a \omega\|u\|_{\infty}^{p-1}+2 b \omega+K \omega+\|e\|_{\infty} \omega\right) \\
& \leq \lambda\left(2 a \omega M_{1}^{p-1}+2 K \omega+2 b \omega+\|e\|_{\infty} \omega\right):=\lambda M_{2}^{\prime} .
\end{aligned}
$$

We claim that there exists a positive constant $M_{2}>M_{2}^{\prime}+1$ such that, for all $t \in \mathbb{R}$,

$$
\left\|u^{\prime}\right\|_{\infty} \leq M_{2}
$$

In fact, if $u^{\prime}$ is not bounded, there exists a positive constant $M_{2}^{\prime \prime}$ such that $\left\|u^{\prime}\right\|_{\infty}>M_{2}^{\prime \prime}$ for some $u^{\prime} \in \mathbb{R}$. Therefore, we have

$$
\begin{aligned}
\left\|\phi_{p}(A u)^{\prime}\right\|_{\infty} & =\left\|\phi_{p}\left(A u^{\prime}\right)\right\|_{\infty}=\left\|A u^{\prime}\right\|_{\infty}^{p-1} \\
& =(1+|c|)^{p-1}\left\|u^{\prime}\right\|_{\infty}^{p-1} \geq(1+|c|)^{p-1} M_{2}^{\prime \prime p-1}:=M_{2}^{*} .
\end{aligned}
$$

Then it is a contradiction. So (3.14) holds. 
On the other hand, it follows by (2.1) that

$$
\left(\phi_{p}(A u)^{\prime}(t)\right)^{\prime}+\lambda f\left(t, u^{\prime}(t)\right)+\lambda\left(g_{0}(u(t))+g_{1}(t, u(t))\right)=\lambda e(t) .
$$

Multiplying both sides of (3.15) by $u^{\prime}(t)$ we get

$$
\begin{aligned}
& \left(\phi_{p}(A u)^{\prime}(t)\right)^{\prime} u^{\prime}(t)+\lambda f\left(t, u^{\prime}(t)\right) u^{\prime}(t)+\lambda\left(g_{0}(u(t))+g_{1}(t, u(t))\right) u^{\prime}(t) \\
& \quad=\lambda e(t) u^{\prime}(t)
\end{aligned}
$$

Let $\tau \in[0, \omega]$ be as in (3.3), for any $\tau \leq t \leq \omega$, we integrate (3.16) on $[\tau, t]$ and get

$$
\begin{aligned}
\lambda \int_{u(\tau)}^{u(t)} g_{0}(v) d v= & \lambda \int_{\tau}^{t} g_{0}(u(s)) u^{\prime}(s) d s \\
= & -\int_{\tau}^{t}\left(\phi_{p}(A u)^{\prime}(s)\right)^{\prime} u^{\prime}(s) d s-\lambda \int_{\tau}^{t} f\left(s, u^{\prime}(s)\right) u^{\prime}(s) d s \\
& -\lambda \int_{\tau}^{t} g_{1}(s, u(s)) u^{\prime}(s) d s+\lambda \int_{\tau}^{t} e(s) u^{\prime}(s) d s
\end{aligned}
$$

By (3.7), (3.11) and (3.14), we have

$$
\begin{aligned}
& \left|\int_{\tau}^{t}\left(\phi_{p}(A u)^{\prime}(s)\right)^{\prime} u^{\prime}(s) d s\right| \\
& \quad \leq \int_{0}^{T}\left|\left(\phi_{p}(A u)^{\prime}(s)\right)^{\prime}\right|\left|u^{\prime}(s)\right| d s \\
& \quad \leq \lambda\left\|u^{\prime}\right\|_{\infty}\left(\int_{0}^{\omega}\left|f\left(t, u^{\prime}(t)\right) d t+\int_{0}^{\omega}\right| g(t, u(t))\left|d t+\int_{0}^{\omega}\right| e(t) \mid d t\right) \\
& \quad \leq \lambda M_{2}\left(K \omega+2 a \omega \mid u\left\|_{\infty}^{p-1}+2 b \omega+K \omega+\right\| e \|_{\infty} \omega\right) \\
& \quad \leq \lambda M_{2}\left(2 K \omega+2 a \omega M_{1}^{p-1}+2 b \omega+\|e\|_{\infty} \omega\right) .
\end{aligned}
$$

Moreover, from $\left(H_{1}\right)$ and (3.14)

$$
\begin{aligned}
& \left|\int_{\tau}^{t} f\left(s, u^{\prime}(s)\right) u^{\prime}(s) d s\right| \leq \int_{0}^{T}\left|f\left(s, u^{\prime}(s)\right)\right|\left|u^{\prime}(s)\right| d s \leq K M_{2} \omega \\
& \left|\int_{\tau}^{t} g_{1}(s, u(s)) u^{\prime}(s) d s\right| \leq \int_{0}^{T}\left|g_{1}(s, u(s))\right|\left|u^{\prime}(s)\right| d s \leq M_{2}\left|g_{M_{1}}\right| \sqrt{\omega}
\end{aligned}
$$

where $g_{M_{1}}=\max _{0 \leq u \leq M_{1}}\left|g_{1}(t, u)\right| \in L^{2}(0, \omega)$.

$$
\left|\int_{\tau}^{t} e(s) u^{\prime}(s) d s\right| \leq \int_{0}^{\omega}|e(s)|\left|u^{\prime}(s)\right| d s \leq\|e\|_{\infty} \omega M_{2}
$$

With these inequalities we can derive from (3.17) that

$$
\left|\int_{u(\tau)}^{u(t)} g_{0}(v) d v\right| \leq M_{2}\left(3 K \omega+2 a \omega M_{1}^{p-1}+2 b \omega+2\|e\|_{\infty} \omega+\left|g_{M_{1}}\right| \sqrt{\omega}\right)
$$


In view of (1.6), we know there exists $M_{3}>0$ such that

$$
u(t) \geq M_{3}, \quad \forall t \in[\tau, \omega] .
$$

The case $t \in[0, \tau]$ can be treated similarly.

Having in mind (3.11), (3.14) and (3.18), we define

$$
\Omega=\left\{u \in X: E_{1}<u(t)<E_{2} \text { and }\left|u^{\prime}(t)\right|<E_{3} \forall t \in \mathbb{R}\right\},
$$

where $0<E_{1}<\min \left\{D_{2}, M_{3}\right\}, E_{2}>\max \left\{M_{1}, D_{1}\right\}$ and $E_{3}>M_{2}$. We know that (2.1) has no solution on $\partial \Omega$ as $\lambda \in(0,1)$ and when $u(t) \in \partial \Omega \cap \mathbb{R}, u(t)=E_{2}$ or $u(t)=E_{1}$, from (3.4), we know that $E_{2}+1>D_{1}$; therefore, from $\left(H_{2}\right)$ we see that

$$
\frac{1}{\omega} \int_{0}^{\omega} g\left(t, E_{2}\right) d t<0
$$

and

$$
\frac{1}{\omega} \int_{0}^{\omega} g\left(t, E_{1}\right) d t>0
$$

So condition (ii) is also satisfied. Set

$$
H(u, \mu)=\mu u+(1-\mu) \frac{1}{\omega} \int_{0}^{\omega} g(t, u) d t
$$

where $x \in \partial \Omega \cap \mathbb{R}, \mu \in[0,1]$, we have

$$
u H(u, \mu)=\mu u^{2}+(1-\mu) \frac{u}{\omega} \int_{0}^{\omega} g(t, u) d t \neq 0
$$

and thus $H(u, \mu)$ is a homotopic transformation and

$$
\begin{aligned}
\operatorname{deg}\{F, \Omega \cap \mathbb{R}, 0\} & =\operatorname{deg}\left\{\frac{1}{\omega} \int_{0}^{\omega} g(t, u) d t, \Omega \cap \mathbb{R}, 0\right\} \\
& =\operatorname{deg}\{u, \Omega \cap \mathbb{R}, 0\} \neq 0 .
\end{aligned}
$$

So condition (iii) is satisfied. In view of Lemma 2.1, there exists a solution with period $\omega$.

\section{Example}

Example 4.1 Consider the following $p$-Laplacian neutral Rayleigh equation with singularity:

$$
\begin{aligned}
& \left(\phi_{p}\left(u(t)-\frac{1}{4} u(t-\delta)\right)^{\prime}\right)^{\prime}-\cos ^{2}(2 t) \sin u^{\prime}(t)-\frac{1}{3 \pi^{4}}(\sin 4 t+2) u^{3}(t)+\frac{1}{u^{\mu}} \\
& \quad=\sin ^{2}(2 t)
\end{aligned}
$$

where $\mu \geq 1$ and $p=4, \delta$ is a constant and $0 \leq \delta<\omega$. 
It is clear that $\omega=\frac{\pi}{2}, c=\frac{1}{4}, e(t)=\sin ^{2}(2 t), f(t, v)=-\cos ^{2}(2 t) \sin v, g(t, u)=-\frac{1}{3 \pi^{3}}(\sin 4 t+$ 2) $u^{4}(t)+\frac{1}{u^{\mu}(t)}$. Choose $K=1, D_{1}=2, D_{2}=1, a=\frac{1}{\pi^{4}}$, it is obvious that $\left(H_{1}\right),\left(H_{2}\right)$ and $\left(H_{3}\right)$ hold. Next, we consider

$$
\begin{aligned}
& \frac{\omega(1+|c|)^{\frac{1}{p}} a^{\frac{1}{p}}}{2^{\frac{p-1}{p}}|1-| c||} \\
& =\frac{\frac{\pi}{2}\left(1+\frac{1}{4}\right)^{\frac{1}{4}}\left(\frac{1}{\pi^{4}}\right)^{\frac{1}{4}}}{2^{\frac{3}{4}}\left(1-\frac{1}{4}\right)} \\
& \approx \frac{1.057}{1.783}<1 .
\end{aligned}
$$

Therefore, by Theorem 3.1, (4.1) has at least one nonconstant $\frac{\pi}{2}$-periodic solution.

\section{Conclusions}

In this article we introduce the existence of a periodic solution for a $p$-Laplacian neutral Rayleigh equation with singularity of attractive type. Due to the attractive condition being in contradiction with the repulsive condition, the methods of $[12,15,16]$ are no long applicable to the proof of a periodic solution for equation (1.5) with singularity of attractive singularity. In this paper, we give attractive conditions (1.6) and $\left(H_{3}\right)$, and we see the existence of a periodic solution for (1.5) by applications of the extension of Mawhin's continuation theorem [17]. Moreover, in view of the mathematical points, the results satisfying the conditions of an attractive singularity are valuable to understand the periodic solution for Rayleigh equations.

\section{Acknowledgements}

$Y X, H M L$ and ZBC would like to thank the referee for invaluable comments and insightful suggestions. This work was supported by National Natural Science Foundation of China (No. 11501170), Education Department of Henan Province project (No. 16B110006) and Henan Polytechnic University Outstanding Youth Fund (J2016-03).

\section{Competing interests}

The authors declare that they have no competing interests.

Authors' contributions

YX, HML and ZBC worked together on the derivation of the mathematical results. All authors read and approved the final manuscript.

\section{Author details}

${ }^{1}$ College of Computer Science and Technology, Henan Polytechnic University, Jiaozuo, China. ${ }^{2}$ School of Mathematics and Information Science, Henan Polytechnic University, Jiaozuo, China. ${ }^{3}$ Department of Mathematics, Sichuan University, Chengdu, China.

\section{Publisher's Note}

Springer Nature remains neutral with regard to jurisdictional claims in published maps and institutional affiliations.

Received: 4 January 2018 Accepted: 7 March 2018 Published online: 14 March 2018

\section{References}

1. Gaines, R.E., Mawhin, J.L.: Coincidence Degree, and Nonlinear Differential Equations. Lecture Notes in Mathematics, vol. 568. Springer, Berlin (1977)

2. Cheng, Z.B., Ren, J.L.: Periodic solutions for a fourth-order Rayleigh type $p$-Laplacian delay equation. Nonlinear Anal. 70, 516-523 (2009)

3. Cheung, W., Ren, J.L.: Periodic solutions for p-Laplacian Rayleigh equations. Nonlinear Anal. 65, 2003-2012 (2006)

4. Cheung, W., Ren, J.L., Han, W.: Positive periodic solution of second-order neutral functional differential equations. Nonlinear Anal. 71, 3948-3955 (2009)

5. Du, B., Lu, S.P.: On the existence of periodic solutions to a $p$-Laplacian Rayleigh equation. Indian J. Pure Appl. Math. 40, 253-266 (2009) 
6. Habets, P., Torres, P.: P: Some multiplicity results for periodic solutions of a Rayleigh differential equation. Dyn. Contin. Discrete Impuls. Syst., Ser. A Math. Anal. 8, 335-351 (2001)

7. Ma, T.: Periodic solutions of Rayleigh equations via time-maps. Nonlinear Anal. 75, 4137-4144 (2012)

8. Wang, L., Shao, J.: New results of periodic solutions for a kind of forced Rayleigh-type equations. Nonlinear Anal., Real World Appl. 11, 99-105 (2010)

9. Wang, Z.H.: On the existence of periodic solutions of Rayleigh equations. Z. Angew. Math. Phys. 56, 592-608 (2005)

10. Wang, Y., Dai, X.: Existence and stability of periodic solutions of a Rayleigh type equation. Bull. Aust. Math. Soc. 79, 377-390 (2009)

11. Xin, Y., Cheng, Z.B.: Existence and uniqueness of a positive periodic solution for Rayleigh type $\phi$-Laplacian equation. Adv. Differ. Equ. 2014, 225 (2014)

12. Chen, L.J., Lu, S.P.: A new result on the existence of periodic solutions for Rayleigh equations with a singularity of repulsive type. Adv. Differ. Equ. 2017, 106 (2017)

13. Lu, S.P., Zhang, T., Chen, L.: Periodic solutions for $p$-Laplacian Rayleigh equations with singularities. Bound. Value Probl. 2016, 96 (2016)

14. Sun, X., Yu, P., Qin, B.: Global existence and uniqueness of periodic waves in a population model with density-dependent migrations and Allee effect. Int. J. Bifurc. Chaos Appl. Sci. Eng. 27, 1750192 (2017)

15. Wang, Z.H., Ma, T.: Periodic solutions of Rayleigh equations with singularities. Bound. Value Probl. 2015, 154 (2015)

16. Xin, Y., Cheng, Z.B.: Study on a kind of neutral Rayleigh equation with singularity. Bound. Value Probl. 2017, 92 (2017)

17. Lu, S.P.: Periodic solutions to a second order $p$-Laplacian neutral functional differential system. Nonlinear Anal. TMA $69,4215-4229$ (2008)

18. Zhang, M.R.: Periodic solutions of linear and quasilinear neutral functional differential equations. J. Math. Anal. Appl. 189, 378-392 (1995)

19. Xin, Y., Cheng, Z.B.: Positive periodic solution of $p$-Laplacian Liénard type differential equation with singularity and deviating argument. Adv. Differ. Equ. 2016, 41 (2016)

\section{Submit your manuscript to a SpringerOpen ${ }^{\circ}$ journal and benefit from:}

- Convenient online submission

- Rigorous peer review

- Open access: articles freely available online

- High visibility within the field

Retaining the copyright to your article

Submit your next manuscript at $\gg$ springeropen.com 